\title{
IMAGE QUALITY ASSESSMENT OF TONE MAPPED IMAGES
}

\author{
Jino Lee, Rae-Hong Park \\ Sogang University, Baekbeom-ro (Sinsu-dong), Mapo-gu, Korea
}

\begin{abstract}
This paper proposes an objective assessment method for perceptual image quality of tone mapped images. Tone mapping algorithms are used to display high dynamic range (HDR) images onto standard display devices that have low dynamic range (LDR). The proposed method implements visual attention to define perceived structural distortion regions in LDR images, so that a reasonable measurement of distortion between HDR and LDR images can be performed. Since the human visual system is sensitive to structural information, quality metrics that can measure structural similarity between HDR and LDR images are used. Experimental results with a number of HDR and tone mapped image pairs show the effectiveness of the proposed method.
\end{abstract}

\section{KEYWORDS}

Objective quality assessment, High dynamic range image, Tone mapping, Visual saliency, Structural fidelity

\section{INTRODUCTION}

Dynamic range (DR) of conventional display devices is on the order of 100 to 1 whereas that of real scene is larger than 10,000 to 1 [1], [2]. Digital still and video cameras have the capability to acquire the DR larger than 100 to 1 to satisfy acceptable quality, with less noise interference and natural contrast representation [2]-[4]. Moreover, to obtain a perceptually high-quality image, consumer digital cameras need to capture real scene as human eyes do. To visualize real scene more accurately, a technique is needed to effectively compress intensity variation of high dynamic range (HDR) image data. Tone mapping operators (TMOs) allow display devices to show HDR images as low dynamic range (LDR) images [1]-[9]. However, converted LDR images using TMOs may lose detail information of HDR images, and have distortions and artifacts because TMOs compress intensity values of HDR images to the limited range of $0-255$, for example.

The human visual system (HVS) is highly responding to stimulus of structural information [10][13]. Therefore, the performance of TMOs in terms of human visual perception can be achieved. Subjective assessment methods of tone mapped images evaluate contrast, details, and brightness of LDR images [5]-[7]. Some of quality attributes used in subjective assessment, such as brightness, contrast, and structural information of images, are also considered in conventional objective assessment methods [14]-[19].

Many objective assessment methods measure structural distortion of images [9], [14]-[19]. Mantiuk et al. proposed visual difference predicator (VDP) for HDR images [9]. HDR-VDP measures contrast difference between reference HDR images and tone mapped images. Aydin et al. proposed improved contrast distortion [15], in which contrast distortions defined as loss, reversal, and amplification of contrast can reflect structural distortion of tone mapped images. Structural similarity index metric (SSIM) was proposed by Wang and Bovik [16]. It extracts structural distortion between reference image and distorted image as the HVS perceives structural information of images. Yeganeh and Wang proposed modified SSIMs for tone mapped images [17]-[19]. Because of the difference of DR between HDR and LDR images, they proposed a DOI : $10.5121 /$ ijcga.2015.5202 
modified metric that can reduce the influence of DR difference. Also, they measured naturallooking quality of tone mapped images as global contrast and brightness with their own naturalness model.

Visual attention models have been widely used in image and video quality assessment to reduce the complexity of scene analysis and to correlate with the HVS [20]-[25]. Visual attention measures local stimuli of images and computes guide perceptual difference between the reference and tone mapped images using perceptual image structure information [20]. The proposed method measures structural distortion between images in highly perceived regions. Subjective quality observer cannot evaluate detail loss of the whole image region in HDR scene [17]-[19]. Thus, objective quality measurement over the entire image can give wrong estimation results. Liu et al. studied extension of SSIM of tone mapped images [19] using several visual saliency methods [25], which shows the effectiveness of visual attention in image quality methods. The proposed method integrates visual attention method to increase awareness of the HVS to image quality assessment of tone mapped image.

The rest of the paper is structured as follows. Section 2 describes perceptual characteristic of the HVS used in the proposed algorithm. In Section 3, we explain the proposed image quality assessment method of tone mapped images. First, a visual saliency measurement method is proposed and then the proposed image quality metric is presented. In Section 4, experimental results are given and discussed. Finally, Section 5 concludes the paper.

\section{Perceptual Distortion Model}

The HVS sensitivity to structural distortion is influenced by local luminance level [11]-[14]. Fig. 1 shows visibility threshold achieved in standard DR (8-bit) as a function of background luminance that is assumed in the range of 0 to 255 . Visibility threshold is used to determine the amount of intensity difference at which human can detect the variation of local luminance levels. Chou and Li showed adaptive visibility threshold values as a function of background luminance [12], as shown in Fig. 1 (solid line). The human eyes are observed to be less sensitive to low luminance region. In addition, according to Weber contrast model, luminance adaptation in higher background luminance region needs larger visibility threshold value. Zhang et al. used Watson's luminance adaptation model using Weber-Fechner's law, which is also shown in Fig. 1 (dashed line) [13].

In Chou and Li's model, small variations and distortions in low luminance region can be regarded as not significant to quality assessment. On the contrary, Weber-Fechner's law shows that human eyes quickly sense low luminance region and can detect image distortions. In high luminance region, human eyes are stimulated to strong luminance variation. This effect is called contrast masking effect [26]. Contrast masking induces human eyes to respond to large contrast variations and to ignore small contrast variations. Local luminance level influences on contrast masking. When local luminance value becomes high, the HVS cannot detect distortions of local image information. In low luminance regions, the HVS can sensitively respond to small contrast variation. 


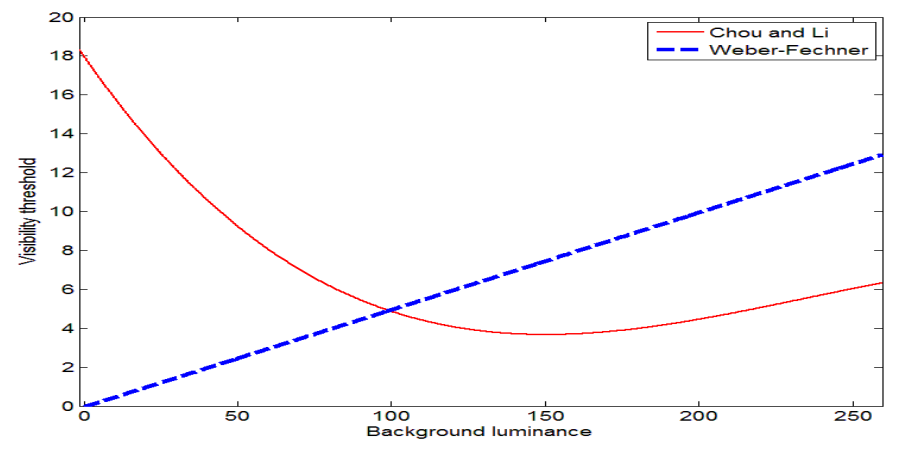

Figure 1. Two adaptive visibility thresholds as a function of background luminance. Chou and Li's nonlinear visibility threshold values (solid line) and Watson's luminance adaptation model using Weber-

Fechner's law (dashed line)

Mantiuk et al. proposed a contrast discrimination model to analyze HDR scenes for tone mapping and contrast enhancement algorithms [27]. Weber contrast fraction model, which measures contrast visibility in LDR scenes well, can incorrectly predict high contrast of HDR scenes. Therefore, they use a logarithmic contrast ratio to measure visibility threshold of HDR scenes.

DR compression may cause loss of detail and structural information. We consider this distortion characteristic in evaluation of tone mapped images. Subjective image evaluation cannot reflect all distortions of tone mapped images. In evaluation of tone mapped images using HDR and LDR scenes, variable visibility thresholds have an effect on recognition of distorted region because background luminance and local contrast of tone mapped LDR images are different from reference HDR scenes. Salient regions have low visibility threshold values in the proposed method, thus observers can detect distortion between the reference scene and tone mapped images. On the contrary, in weak visual attention regions, strong contrast variations affect perception of distortions. An objective image quality measure in high visibility threshold regions may not affect subjective quality assessment. Thus, the proposed method detects high salient regions using HVS characteristics that are related to local luminance level. The performance of the quality measure can be degraded because it uses the mean value of local (pixel-wise) quality scores [15]-[19]. Thus, the proposed method focuses on quality measure of perceptible distortion regions. To detect regions of non-visible distortion that can influence the performance of the quality measure, we consider only strong structural information in weak salient regions in the proposed image quality assessment.

\section{Proposed Image Quality Assessment}

The proposed method consists of two steps: visual saliency measure and quality measurement. Fig. 2 shows the block diagram of the proposed method. With a visual attention prediction method, the proposed method first detects interest regions of a reference image $x$ and tone mapped image $y$. In quality measurement step, a weight map is used with saliency maps of $x$ and $y$ to obtain overall quality score, which is highly correlated with subjective evaluation of tone mapped images. A weight map is combined with two saliency maps of reference and tone mapped images to measure attention image region in both images, $x$ and $y$. Detailed description of each step is given in the following 


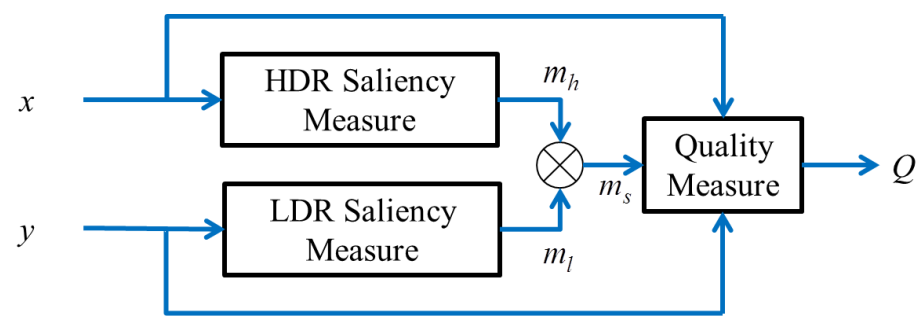

Figure 2. Block diagram of the proposed image quality assessment method of tone mapped images

\subsection{Visual Saliency Map}

Conventional saliency measurement methods have some drawbacks to integrate quality assessment of tone mapped images because they focus on standard DR [17]. Most visual attention approaches lack of considering dynamic range of image features, thus conventional methods give poor visual attention information in HDR images. To achieve better saliency prediction result of HDR scenes, the proposed method extends saliency measurement method. The proposed method uses contrast information of HDR images in saliency measurement in HDR saliency measure.

Fig. 3 shows a visual saliency map of a HDR image. Perceptual structure information in the reference image (HDR image) $x$ is used to consider structure loss in highlight regions of a tone mapped image $y$. The proposed methods consider visual contrast variation with saliency map to detect perceived distortions between HDR and tone mapped images.

Commonly, saliency estimation methods use difference of luminance values [28], [29]. However, the HVS responds to local (background) luminance values, rather than pixel-wise values. For better saliency detection results with visual adaptation, difference of intensity contrast is used [29]. Bremond et al. [29] modified Itti et al.'s saliency measurement [28] of HDR image. Itti et al.'s method estimates saliency of image in image intensity domain, however, Bremond et al.'s method estimates saliency image in image contrast domain. Two saliency features of their methods $I$ and $O$ are modified as

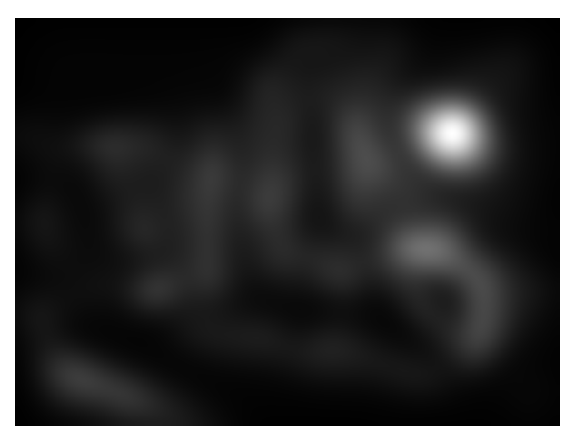

(a)

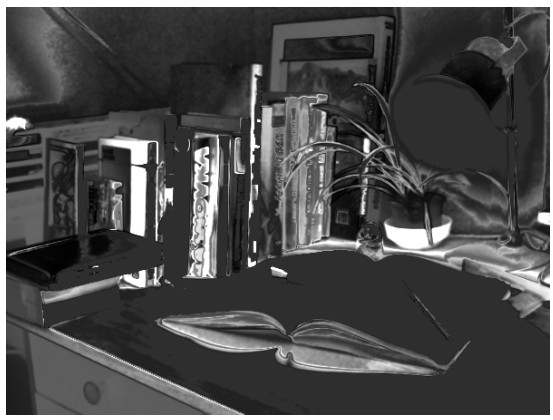

(b)

Figure 3. Visual saliency map of a HDR image, (a) Bremend et al.'s method [28], (b) Achanta et al.'s method [29]

$$
I^{\prime}\left(s_{1}, s_{2}\right)=\frac{\left|I\left(s_{1}\right)-I\left(s_{2}\right)\right|}{I\left(s_{2}\right)}
$$


where $s_{1}$ and $s_{2}$ are different two scales. Orentation feature is defined as

$$
O^{\prime}\left(s_{1}, s_{2}, \theta\right)=\frac{O\left(s_{1}, \theta\right)}{I\left(s_{2}\right)}
$$

where $\theta$ is the orientation of a Gabor filter. Saliency of the target image is combined with multiscaled Gabor filter. Result of saliency estimation is in Fig. $3 a$ which shows saliency region roughly. Achanta et al. proposed the full-resolution saliency map using background luminance [30], which is expressed as

$$
M_{S}(i, j)=\left\|\mathbf{I}_{\mu}(i, j)-\mathbf{I}_{g}(i, j)\right\|
$$

where $\mathbf{I}_{\mu}$ represents a mean image feature vector and $\mathbf{I}_{g}$ denotes a Gaussian blurred image vector that are estimated using an $11 \times 11$ kernel. Fig. $3 b$ shows a saliency map by Achanta et al.'s method that shows more detail information.

Multi-scaled filter based saliency map represents saliency region well, however, frequency and object information are not faithfully described. Moreover, the proposed method employs multiscale SSIM [32], and attributes of saliency across image scale in Fig. $3 a$ can be accompanied with simple saliency detection method. Liu et al. used visual saliency of LDR images only [25]. However, the proposed method combines HDR and LDR visual attentions. The proposed method adapts Achanta et al.'s method to measure saliency maps of HDR image and tone mapped image, $m_{h}$ and $m_{l}$, which are defined as

$$
\begin{gathered}
m_{h}(i, j)=\left\|\mathbf{X}_{\mu}(i, j)-\mathbf{X}_{g}(i, j)\right\|, \\
m_{l}(i, j)=\left\|\mathbf{Y}_{\mu}(i, j)-\mathbf{Y}_{g}(i, j)\right\|
\end{gathered}
$$

where $\mathbf{X}$ and $\mathbf{Y}$ represent Yxy colour-space images of $x$ and $y$, respectively. Saliency-based weight map $m_{s}^{l}$ of each pyramid level $l$ is simply obtained as

$$
m_{s}^{l}(i, j)=m_{l}^{l}(i, j) \times m_{h}^{l}(i, j) .
$$

\subsection{QUALITY MEASURES}

To measure the structural fidelity between reference and distorted images, local SSIM was proposed by Wang and Bovik [16]. Using luminance, contrast, and structure terms, the SSIM is defined as multiplication of three terms:

$$
\operatorname{SSIM}(x, y)=\frac{2 \mu_{x} \mu_{y}+C_{1}}{\mu_{x}^{2}+\mu_{y}^{2}+C_{1}} \cdot \frac{2 \sigma_{x} \sigma_{y}+C_{2}}{\sigma_{x}^{2}+\sigma_{y}^{2}+C_{2}} \cdot \frac{2 \sigma_{x y}+C_{3}}{\sigma_{x} \sigma_{y}+C_{3}}
$$


where $x$ represents a reference image and $y$ denotes a degraded image. $\mu$ and $\sigma$ indicate Gaussian weighted filtered average and standard deviation, respectively, and $\sigma_{x y}$ signifies covariance of $x$ and $y . C_{1}, C_{2}$, and $C_{3}$ are non-zero constants to avoid instability. Luminance (first term) measures the similarity of local averaged luminance value. Contrast (second term) measures the similarity of local variation whereas structure (third term) measures the cross correlation between $x$ and $y$.

Yeganeh and Wang proposed a modified structural fidelity for quality measure of tone mapped images with a contrast mapping function [17]-[19]. The modified SSIM [17], [18] is defined as

$$
\operatorname{SSIM}^{\prime}(x, y)=\frac{2 \sigma_{x}^{\prime} \sigma_{y}^{\prime}+C_{2}}{\sigma_{x}^{\prime 2}+\sigma_{y}^{\prime 2}+C_{2}} \cdot \frac{2 \sigma_{x y}+C_{3}}{\sigma_{x} \sigma_{y}+C_{3}}
$$

where $\sigma^{\prime}$ is obtained by a non-linear mapping function defined as

$$
\sigma_{x}^{\prime}= \begin{cases}0, & \frac{1}{2}\left\{1+\cos \left[\frac{\pi}{T_{2}-T_{1}}\left(\sigma_{x}-T_{2}\right)\right]\right\}, \\ 1, & T_{1} \leq \sigma_{x} \leq T_{2} . \\ & T_{2}<\sigma_{x}\end{cases}
$$

Threshold values $T_{1}$ and $T_{2}$ decide significant contrast range. The modified SSIM does not use luminance term (first term in (7)) because DRs of $x$ and $y$ are much different from each other in computing the quality measure of tone mapped images. Overall structural fidelity score $Q_{Y W}$ of Yeganeh and Wang's method is defined as

$$
Q_{Y W}=\frac{\sum_{i=1}^{W} \sum_{j=1}^{H} \operatorname{SSIM}^{\prime}(i, j)}{W \times H}
$$

where $W$ and $H$ represent width and height of a tone mapped image, respectively.

In [19], an HVS based non-linear mapping function is used to penalize the DR difference between HDR and LDR scenes. The DR difference is defined as

$$
\sigma_{x}^{\prime}=\frac{1}{\sqrt{2 \pi} \theta_{\sigma}} \int_{-\infty}^{\sigma} \exp \left[-\frac{\left(x-\tau_{\sigma}\right)^{2}}{2 \theta_{\sigma}^{2}}\right] d x
$$

where $\tau_{\sigma}$ represents a modulation threshold and $\theta_{\sigma}$ denotes a scale parameter of the normal function.

The proposed method also uses contrast and structure terms in (7) without luminance term, which is expressed as 


$$
S(x, y)=\frac{2 \sigma_{x} \sigma_{y}+C_{2}}{\sigma_{x}^{2}+\sigma_{y}^{2}+C_{2}} \cdot \frac{2 \sigma_{x y}+C_{3}}{\sigma_{x} \sigma_{y}+C_{3}} .
$$

The proposed method uses the entire range of contrast values because (8) may not reflect perceptive contrast information of $x$ and $y$. Variation of contrast is noticeable to observers, however, (9) limits contrast dissimilarity between $x$ and $y$ and thus cannot be said to consider luminance adaptation.

The proposed method penalizes DR difference with saliency map $m$ [25]. A quality score of a tone mapped image of the proposed image quality assessment is defined as

$$
Q=\frac{1}{W \times H} \frac{\sum_{i=1}^{W} \sum_{j=1}^{H} S(i, j) \cdot m_{s}(i, j)}{\sum_{i=1}^{W} \sum_{j=1}^{H} m_{s}(i, j)} .
$$

We previously described visual saliency measurement in Section 3.1 for decision of visible distortion region. Using (6), the proposed method calculates local similarity at pixel with $m_{s}=1$ and quality score of a tone mapped image is computed as the mean value of their local similarities. The proposed method uses multi-scale SSIM (MSSSIM) [31] to verify the performance of the proposed method with the same parameter setting in [17]-[19]. Quality map of each scale $l$ is defined as

$$
Q^{l}=\frac{\sum_{i=1}^{W^{l}} \sum_{j=1}^{H^{l}} S^{l}(i, j) \cdot m_{s}^{l}(i, j)}{\sum_{i=1}^{W^{l}} \sum_{j=1}^{H^{l}} m_{s}^{l}(i, j)} .
$$

The quality score is defined as

$$
Q=\prod_{l=1}^{L} Q_{l}^{\beta^{l}}
$$

where $L=5$ and $\beta^{l}=\{0.0448,0.2856,0.3001,0.2363,0.1333\}$.

Fig. 4 compares pixel-wise quality scores obtained by quality metrics (8) and (12). Fig. $4 a$ is a quality map using (8), which shows that a non-linear mapping function (9) limits similarity comparison in some regions of tone mapped images. Fig. $4 b$ is a quality map with contrast mapping function (11), which shows better performance than Fig. 4a. Fig. $4 c$ shows pixel-wise quality scores of the proposed quality metric that shows distinct comparison result with visual saliency characteristics. 


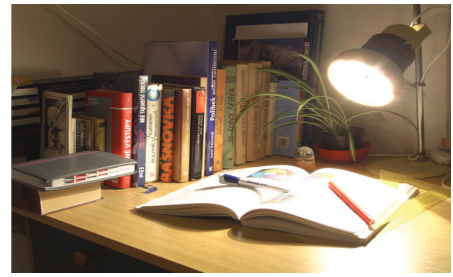

(a)

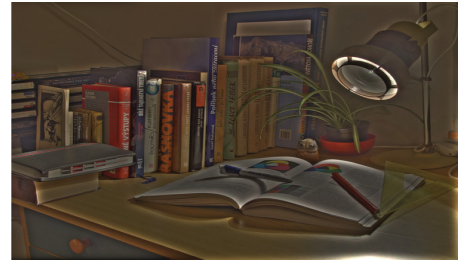

(d)

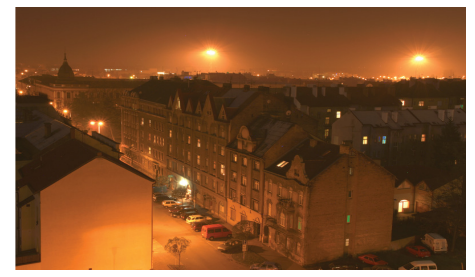

(b)

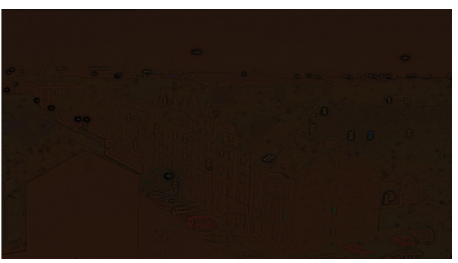

(e)

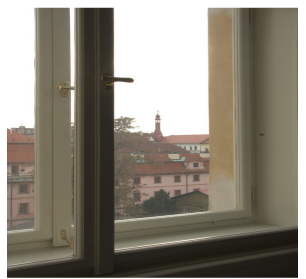

(c)

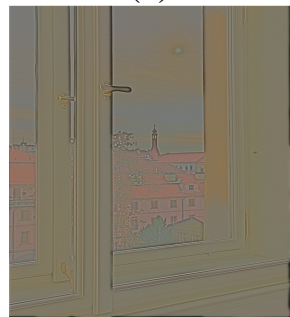

(f)

Figure 5. Comparison of structural fidelity scores with test images. (a), (b), (c) tone mapped images using linear clipping method [5] (rank 1) (d), (e), (f) tone mapped images using Chiu et al.'s method [10] (rank 14).

\section{EXPERIMENTAL RESULTS AND DISCUSSIONS}

We compare the performance of the proposed method, Yeganeh and Wang's objective evaluation methods [18], [19], and Liu et al.'s method [25]. Cadik et al.'s subjective evaluation data [5], in which 11 observers evaluated three HDR scenes with 14 tone mapping algorithms, is used to verify the performance of the proposed method. 14 tone mapping algorithms consist of 5 global and 9 local tone mapping approaches. For verification of the performance of conventional objective assessment methods, mean opinion scores (MOS) can be used to compare subjective scores and objective scores [15], [31]. However, subjective evaluation results of tone mapped images are not enough to construct MOS database. On the other hand, we can compare the performance of image quality metrics using rank similarity between objective scores and subjective scores [15]-[19], [31]. The purpose of objective quality assessments is to help finding best perceptual quality images or image processing algorithms in the given datasets. Therefore, good rank correlation is an important factor in objective image assessment.

We compare rank order correlation coefficients, Spearman and Kendall [32], [33], between two objective assessment methods and subjective ranking. Rank correlation coefficient shows the similarity between objective assessment quality and subjective quality of tone mapped images. High correlation with subjective ranks represents that the performance of quality metrics clearly evaluate high- or low-quality of tone mapped images, in which objective rank concurs well with subjective evaluation results.

Fig. 5 and Table 1 show the performance of objective quality scores against subjective ranking to visually compare the rank correlativity. Fig. 5 shows tone mapped images with two tone mapping methods, where Figs. $5 a, 5 c$, and $5 e$ are tone mapped images using the top-ranked (rank 1) TMO [5] among 14 TMOs compared whereas Figs. 5b, $5 d$, and $5 f$ are those using the bottom-ranked (rank 14) TMO [10]. Table 1 shows the performance of quality metrics with structural fidelity scores of tone mapped images in Fig. 5. Figs. $5 b, 5 d$, and $5 f$ show unnatural local contrast and brightness reproduction. However, overall structural fidelity scores $Q_{Y W}$ of these images are similar to those of Figs. $5 a, 5 c$, and $5 e$, which show natural tone mapping results. Especially, $Q_{Y W}$ 
in Figs. $5 c$ and $5 d$ shows the worst case of Yeganeh and Wang's method. Though Fig. $5 d$ shows poor tone mapping result, quality score of Fig. $5 d$ is higher than that of Fig. $5 c$, which shows that Fig. $5 c$ is better than Fig. $5 d$. Structural fidelity scores $Q$ in Fig. 5 shows the performance of the proposed method. Quality scores of the proposed method in Figs. $5 c$ and $5 d$, which give rank reversal in terms of $Q_{Y W}$, show better correlation with subjective rank. Other two test results in the first and third rows of the proposed method also show better performance than Yeganeh and Wang's method in terms of the rank correlativity with subjective rank.

Table 1. Comparison of structural fidelity scores of tone mapped images in Fig. 5

\begin{tabular}{|c|c|c|c|c|}
\hline & $\boldsymbol{Q}$ & $\boldsymbol{Q}_{Y W}[\mathbf{1 8}]$ & $\boldsymbol{Q}_{Y W}[\mathbf{1 9}]$ & $\boldsymbol{Q}_{V}[\mathbf{2 5}]$ \\
\hline Desk (linear clipping [5]) & $\mathbf{0 . 9 5 7 0}$ & 0.7827 & 0.9619 & 0.9574 \\
\hline Desk (Chiu et al.'s method [10]) & $\mathbf{0 . 2 9 7 7}$ & 0.6417 & 0.7375 & 0.7624 \\
\hline Night (linear clipping [5]) & $\mathbf{0 . 7 4 7 5}$ & 0.8309 & 0.7770 & 0.7752 \\
\hline Night (Chiu et al.'s method [10]) & $\mathbf{0 . 3 1 4 7}$ & 0.7539 & 0.3262 & 0.3426 \\
\hline Window (linear clipping [5]) & $\mathbf{0 . 8 8 3 2}$ & 0.9430 & 0.8476 & 0.8198 \\
\hline Window (Chiu et al.'s method [10]) & $\mathbf{0 . 2 6 4 5}$ & 0.8002 & 0.5354 & 0.6245 \\
\hline
\end{tabular}

Fig. 6 shows scatter plots of 14 tone mapping algorithms according to subjective rank. Overall structural fidelity score $Q_{Y W}$ is the average value of pixel-wise quality scores, hence Yeganeh and Wang's structural fidelity scores are higher than those of the proposed method. However, $Q_{Y W}$ has visually similar scores for each tone mapping algorithm. Fig. 4 can explain the difference of the performance between $Q$ and $Q_{Y W}$ in Fig. 6. Fig. $4 a$ shows the side-effect of non-linear mapping function (9), which limits significant contrast range. Contrast distortions, which are out of threshold range in (9), are regarded as similar structural information as shown in Fig. 4a. The proposed quality metric (12) can measure wider range of distortion than (8). Thus, structural fidelity score differences of the proposed method between high and low ranked TMOs are larger than those of Yageneh and Wang's method.

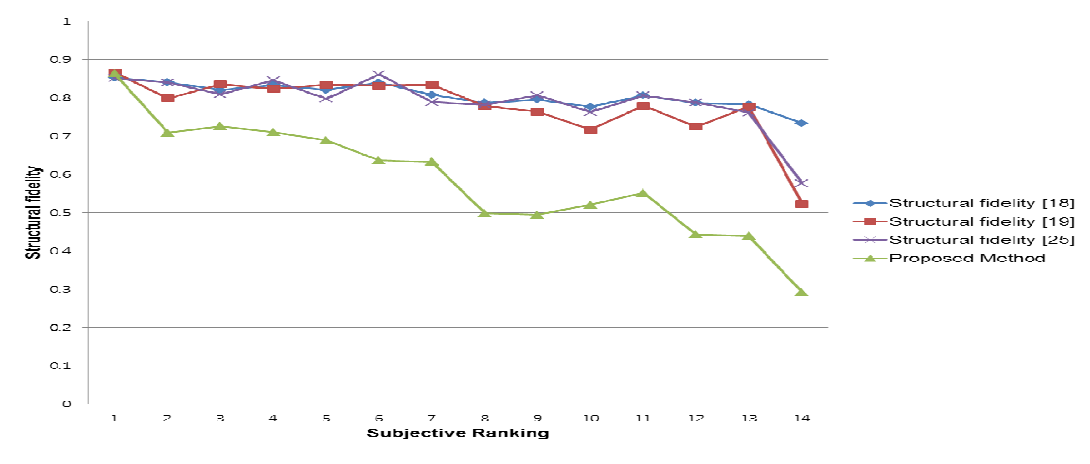

Figure 6. Comparison of quality metrics with structural fidelity scores of tone mapped images

Experimental results in Fig. 7 and Table 2 compare rank correlations between subjective ranking and objective quality scores. Fig. 7 shows performance comparison of four quality measures in terms of the SRCC and KRCC: $Q$, overall quality in [18], [19], and the best performance result $Q_{V}$ in [25] with Zhang et al.'s visual saliency method [34]. The overall quality of Yeganeh and Wang's methods additionally evaluate global brightness and contrast of a tone mapped image and 
combine them with $Q_{Y w}$. The SRCC and KRCC of five quality measures in Fig. 7 are listed in Table 2. Bold numbers signify the best measure for each rank correlation coefficient. In terms of the rank correlation coefficient, the proposed method shows the best performance. Rank correlations of the proposed quality metric (12) are higher than those of Yeganeh and Wang's quality metric (8). Better quality assessments are obtained results with perceptual weight function using saliency map than those of a heuristic function in (9) and Liu et al.'s method.

Yeganeh and Wang's objective quality rank is less correlated with subjective rank than that of the proposed method as shown in Fig. 7 and Table 2. Average structural fidelity scores of the proposed method in Fig. 7 are lower than those of Yeganeh and Wang's method and Liu et al.'s method because we consider variations of the whole contrast range. However, the rank of the proposed method has better correlation with the subjective rank. With perceptual approaches according to luminance adaptation, the proposed method achieves good performance in terms of the rank correlation coefficients with subjective evaluation rank.

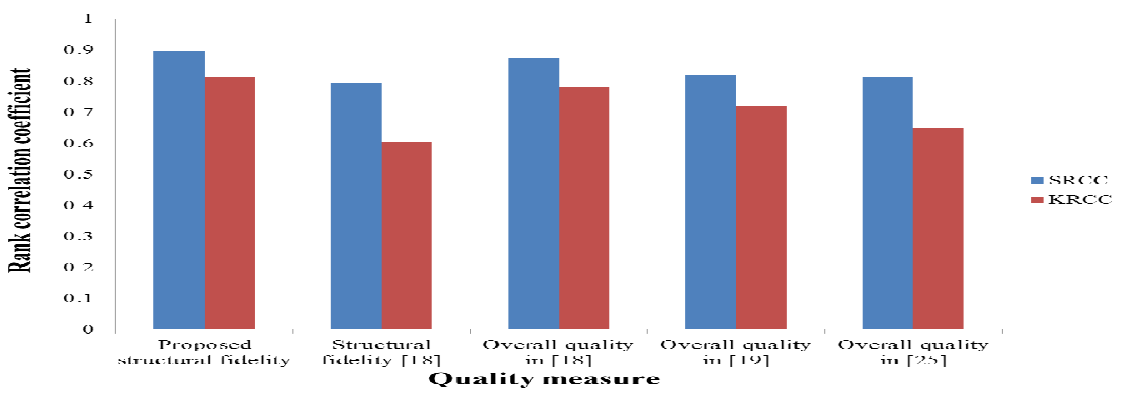

Figure 7. Comparison of quality metrics with the rank correlation coefficients of tone mapped images

Table 2. Comparison of the performance of structural fidelity in terms of the rank order correlation coefficient

\begin{tabular}{|c|c|c|}
\hline & SRCC & KRCC \\
\hline Proposed structural fidelity & 0.8969 & 0.8143 \\
\hline Structural fidelity [18] & 0.7934 & 0.6044 \\
\hline Overall quality in [18] & 0.8759 & 0.7808 \\
\hline Overall quality in [19] & 0.8197 & 0.7179 \\
\hline Overall quality in [25] & 0.8144 & 0.6484 \\
\hline
\end{tabular}

Table 3 shows computation times of Yeganeh and Wang's method, Liu et al.'s method, and the proposed method with $600 \times 450$ image. The computation time is measured by the average of five runs with 14 tone mapped images on a $2.67 \mathrm{GHz}$ CPU, 4GB RAM desktop. The proposed method has higher computational time than Yeganeh and Wang's method [18] (by 16\%), however the performance of the proposed method is better.

Table 3. Comparison of the computation time

\begin{tabular}{|c|c|c|c|c|}
\hline & Proposed & {$[\mathbf{1 8}]$} & {$[\mathbf{1 9 ]}$} & {$[\mathbf{2 5}]$} \\
\hline $\begin{array}{c}\text { Computation time } \\
\text { (seconds) }\end{array}$ & 0.71 & 0.63 & 0.72 & 0.76 \\
\hline
\end{tabular}




\section{CONCLUSIONS}

This paper proposes an improved objective image quality assessment method of tone mapped images. The proposed method uses a modified SSIM quality metric with visual attention in image quality assessment to increase the robustness of DR difference between the reference image and tone mapped images. It integrates visual saliency method to consider the HVS for image quality assessment of tone mapped images. It evaluates how well retaining structural information of distortion of interest regions in each tone mapped image. Experimental results show that the proposed method gives better correlation with subjective evaluation result than Yageneh and Wang's quality assessment method. Further research will focus on the improvement of the weight map to reflect perceptual visibility threshold more accurately and on the consideration of colour naturalness measurement of tone mapped images.

\section{ACKNOWLEDGEMENTS}

This work was supported in part by the Brain Korea 21 Project.

\section{REFERENCES}

[1] E. Reinhard, G. Ward, P. Debevec, and S. Pattanaik, High Dynamic Range Imaging: Acquisition, Display, and Image Based Lighting. Morgan Kaufmann: San Francisco, CA, 2005.

[2] J. Lee, G. Jeon, and J. Jeong, "Piecewise tone reproduction for high dynamic range imaging," IEEE Trans. Consumer Electronics, vol. 55, no. 2, pp. 911-918, May 2009.

[3] Y. Monobe, H. Yamashita, T. Kurosawa, and H. Kotera, "Dynamic range compression preserving local image contrast for digital video camera," IEEE Trans. Consumer Electronics, vol. 51, no. 1, pp. 1-10, Feb. 2005.

[4] S. Cvetkovic, J. Klijn, and P. H. N. With, "Tone-mapping functions and multiple-exposure techniques for high dynamic-range images," IEEE Trans. Consumer Electronics, vol. 54, no. 2, pp. 904-911, May 2008.

[5] M. Cadík, M. Wimmer, L. Neumann, and A. Artusi, "Evaluation of HDR tone mapping methods using essential perceptual attributes," Computers \& Graphics, vol. 32, pp. 330-349, Jun. 2008.

[6] F. P. Ledda, A. Chalmers, T. Troscianko, and H. Seetzen, "Evaluation of tone mapping operators using a high dynamic range display," ACM Trans. Graphics, vol. 24, no. 3, pp. 640-648, Jul. 2005.

[7] A. Yoshida, V. Blanz, K. Myszkowski, and H.-P. Seidel, "Perceptual evaluation of tone mapping operators with real-world scenes," in Proc. Human Vision and Electronic Imaging X, San Jose, CA, vol. 5666, pp. 192-203, Jan. 2005.

[8] P. Choudhury and J. Tumblin, "The trilateral filter for high contrast images and meshes," in Proc. Eurographics Symp. Rendering, Leuven, Belgium, pp. 186-196, Jun. 2003.

[9] R. Mantiuk, K. Myszkowski, and H.-P. Seidel, "Visible difference predicator for high dynamic range images," in Proc. Int. Conf. Systems, Man, and Cybernetics, Hague, Netherlands, pp. 2763-2769, Oct. 2004.

[10] K. Chiu, M. Herf, P. Shirley, S. Swamy, C. Wang, and K. Zimmerman, "Spatially nonuniform scaling functions for high contrast images," in Proc. Graphics Interface, Toronto, Ontario, Canada, pp. 245253, May 1993.

[11] C. F. Hall and E. L. Hall, "A nonlinear model for the spatial characteristics of the human visual system,” IEEE Trans. Systems, Man, and Cybernetics, vol. 7, no. 3, pp. 161-170, Mar. 1977.

[12] C.-H. Chou and Y.-C. Li, "A perceptually tuned subband image coder based on the measure of justnoticeable-distortion profile," IEEE Trans. Circuits and Systems for Video Technology, vol. 5, no. 6, pp. 467-476, Dec. 1995.

[13] X. H. Zhang, W. S. Lin, and P. Xue, "Improved estimation for just-noticeable visual distortion," Signal Processing, vol. 85, no. 4, pp. 795-808, Apr. 2005.

[14] Y. Jia, W. Lin, and A. A. Kassim, "Estimating just-noticeable distortion for video," IEEE Trans. Circuits and Systems for Video Technology, vol. 16, no. 7, pp. 820-829, Jul. 2006. 
[15] T. Aydin, R. Mantiuk, K. Myszkowski, and H. Seidel, "Dynamic range independent image quality assessment," ACM Trans. Graphics, vol. 27, no. 3, pp. 1-10, Aug. 2008.

[16] Z. Wang and A. C. Bovik, "Image quality assessment: From error visibility to structural similarity," IEEE Trans. Image Processing, vol. 13, no. 4, pp. 600-612, Apr. 2004.

[17] H. Yeganeh and Z. Wang, "Objective assessment of tone mapping algorithms," in Proc. Int. Conf. Image Processing, Hong Kong, China, pp. 2477-2480, Sep. 2010.

[18] H. Yeganeh and Z. Wang, "Structural fidelity vs. naturalness - Objective assessment of tone mapped images," in Proc. Int. Conf. Image Analysis and Recognition, Burnaby, BC, Canada, pp. 111-121, Jun. 2011.

[19] H. Yeganeh and Z. Wang, "Objective quality assessment of tone mapped images," IEEE Trans. Image Processing, vol. 22, no. 2, pp. 657-667, Feb. 2013.

[20] U. Engelke, H. Kaprykowsky, H. Zepernick, and P. Y. Ndjiki-Nya, "Visual attention in quality assessment," Signal Processing Magazine, vol. 28, no. 6, pp. 50-59, Nov. 2011.

[21] G. Anan, Z. Debin, L. Shahoui, F. Xiaopeng, and W. Gao, "Visual attention based image quality assessment," in Proc. Image Processing, Brussels, Belgium, pp. 3297-3300, Sep. 2011.

[22] M. C. Q. Faris and W. Y. L Akamine, "On performance of image quality metrics enhanced with visual attention computational models," Electron Letters, vol. 48, no. 11, pp. 631-633, May 2012.

[23] D. Culibrk, M. Mirkovic, V. Zlokolica, M. Pokric, V. Crnojevic, and D. Kukolj, "Salient motion features for video quality assessment," IEEE Trans. Image Processing, vol. 20, no. 4, pp. 948-958, Apr. 2011.

[24] G. Ginesu, F. Massidda, and D. Giusto, "A multi-factors approach for image quality assessment based on a human visual system model," Signal Processing: Image Communication, vol. 21, no. 4, pp. 316333, Apr. 2006.

[25] X. Liu, L. Zhang, H. Li, and J. Lu, "Integrating visual saliency information into objective quality assessment of tone-mapped images", in Proc. Int. Conf. Intelligent Computing, Taiyuan, China, pp. 376-386, Aug. 2014.

[26] G. E. Legge and J. M. Foley, "Contrast masking in human vision," J. Optical Society of America, vol. 70, no. 12, pp. 1458-1470, Dec. 1980.

[27] R. Mantiuk, K. Miszkowski, and H.-P. Siedel, "A perceptual framework for contrast processing of high dynamic range images,” ACM Applied Perception, vol. 3, no. 3, pp. 286-308, Jul. 2006.

[28] L. Itti, C. Koch, and E. Niebur, "A model of saliency-based visual attention for rapid scene analysis," IEEE Trans. Pattern Anal. Machine Intelligence, vol. 20, no. 11, pp. 1254-1259, Nov. 1998.

[29] R. Bremond, J. Petit, and J.-P. Tarel, "Saliency maps of high dynamic range images," in Proc. European Conference on Trends and Topics in Computer Vision, Krete, Greece, pp. 118-130, Sep. 2010.

[30] R. Achanta, S. Hemami, F. Estrada, and S. Susstrunk, "Frequency-tuned salient region detection," in Proc. Computer Vision and Pattern Recognition, Miami, FL, pp. 1597-1604, Jun. 2009.

[31] Z. Wang, E. P. Simoncelli, and A. C. Bovik, "Multi-scale structural similarity for image quality assessment," in Proc. 37th Asilomar Conf. Signals, Systems and Computers, Pacific Grove, CA, pp. 1398-1402, Nov. 2003.

[32] M.-J. Chen and A. C. Bovik, "Fast structural similarity index algorithm," J. Real-Time Image Processing, vol. 6, no. 4, pp. 281-287, Aug. 2011.

[33] M. G. Kendall, “A new measure of rank correlation,” Biometrika, vol. 30, nos. 1/2, pp. 81-93, Jun. 1938.

[34] L. Zhang, Z. Gu, and H. Li, "SDSP: A novel saliency detection method by combining simple priors", in Proc. Int. Conf. Image Processing, Melbourne, Australia, pp. 171-175, Sep. 2013. 